\title{
Coding of Apparent Motion in the Thalamic Nucleus of the Rat Vibrissal Somatosensory System
}

\author{
Valérie Ego-Stengel, ${ }^{\star}$ Julie Le Cam, ${ }^{\star}$ and Daniel E. Shulz \\ Unité de Neuroscience, Information et Complexité, UPR CNRS 3293, Centre National de la Recherche Scientifique, 91198 Gif-sur-Yvette, France
}

While exploring objects, rats make multiple contacts using their whiskers, thereby generating complex patterns of sensory information. The cerebral structures that process this information in the somatosensory system show discrete patterns of anatomically distinct units, each corresponding to one whisker. Moreover, the feedforward and feedback connections are remarkably topographic, with little crosswhisker divergence before reaching the cortical network. Despite this parallel design, information processing from several whiskers has been reported in subcortical nuclei. Here, we explored whether sensory neurons in the ventral posterior medial nucleus (VPM) of the thalamus encode emergent properties of complex multiwhisker stimulations. Using a 24-whisker stimulator, we tested the responses of VPM neurons to sequences of caudal deflections that generated an apparent motion in eight different directions across the whiskerpad. Overall, $45 \%$ of neurons exhibited an evoked increase in firing rate significantly selective to the direction of apparent motion of the global stimulus. Periods of suppression of firing rate were often observed, but were generally not selective. Global motion selectivity of VPM neurons could occur regardless of the extent and spatial organization of their receptive fields, and of their selectivity for the direction of motion of their principal whisker. To investigate whether the global selectivity could be due to corticothalamic feedback connections, we inactivated the barrel cortex while repeating the stimulation protocol. For most VPM neurons, the direction selectivity decreased but was still present. These results suggest that nonlinear processing of stimuli from different whiskers emerges in subcortical nuclei and is amplified by the corticofugal feedback.

\section{Introduction}

Sensory systems are generally organized in a highly topographic manner, reflecting the spatial arrangement of sensory receptors in the periphery. The ventral posterior medial nucleus (VPM) of the thalamus in rodents processes information from the large whiskers on the animal snout (Waite, 1973). The VPM is formed of distinct anatomical aggregates, the barreloids (Van Der Loos, 1976; Williams et al., 1994), corresponding in a one-to-one fashion to the whiskers on the snout. The punctuate organization of the vibrissal system could suggest that the tactile information from single whiskers is processed in parallel. However, when a rat explores an object, multiple whiskers simultaneously contact its surface. Behavioral studies have demonstrated that the CNS can extract high-order features from this spatially distributed stimuli,

Received July 29, 2011; revised Dec. 16, 2011; accepted Dec. 30, 2011.

Author contributions: V.E.-S., J.L.C., and D.E.S. designed research; V.E.-S. and J.L.C. performed research; V.E.-S. contributed unpublished reagents/analytic tools; V.E.-S. and J.L.C. analyzed data;V.E.-S. and D.E.S. wrote the paper.

This work was supported by Agence Nationale pour la Recherche (NATACS and TRANSTACT); European Union Sixth Framework Programme under the grant no. 15879 (FACETS), and European Union Seventh Framework Programme (FP7/2007-2013) under grant agreement no. 243914 (Brain-i-Nets) and no. 269921 (BrainScaleS); and by the Human Frontiers Science Program Organization (Career Development Award to V.E.-S.). We thank Gérard Sadoc, Guillaume Hucher, and Aurélie Daret for technical expertise; Luc Estebanez for help with the surprise method; and Vincent Jacob and Charlotte Deleuze for helpful comments on the manuscript.

*V.E.-S. and J.L.C. contributed equally to this work.

Correspondence should be addressed to Valérie Ego-Stengel, Unité de Neuroscience, Information et Complexité (UNIC), UPR CNRS 3293, Centre National de la Recherche Scientifique, 1 Avenue de la Terrasse, Building 33, 91198 Gif-sur-Yvette, France. E-mail: Valerie.Stengel@unic.cnrs-gif.fr.

DOI:10.1523/JNEUROSCI.3890-11.2012

Copyright $\odot 2012$ the authors $\quad 0270-6474 / 12 / 323339-13 \$ 15.00 / 0$ namely, features that cannot be retrieved if signals from individual whiskers are processed separately (Carvell and Simons, 1995; Krupa et al., 2001; Knutsen et al., 2006). However, at the thalamic level, the capacity of neurons to extract complex information across multiple whiskers remains poorly known.

Functional studies now agree that VPM neurons do receive information from multiple whiskers. In both extracellular (Ito, 1988; Simons and Carvell, 1989; Armstrong-James and Callahan, 1991; Diamond et al., 1992b; Nicolelis and Chapin, 1994; Minnery and Simons, 2003; Timofeeva et al., 2004; Aguilar and Castro-Alamancos, 2005) and intracellular (Chiaia et al., 1991; Brecht and Sakmann, 2002) recordings, the input from one particular whisker, commonly termed the principal whisker of the neuron, strongly dominates. Anatomically, this input would come from only one axon or a few at most, ascending from the brainstem principal nucleus $(\mathrm{PrV})$ of the trigeminal complex (Castro-Alamancos, 2002a; Deschênes et al., 2003; Arsenault and Zhang, 2006). Excitatory responses from surround whiskers are characterized by smaller amplitudes and longer latencies, and could reach the VPM via trigeminothalamic or corticothalamic fibers, the only two sources of excitatory input. Additionally, VPM neurons receive inhibitory projections from the nucleus reticularis (nRt), which carries both principal and surround whiskers information (Lee et al., 1994). By integrating these spatially extended inputs, VPM neurons could analyze tactile properties distributed over several whiskers. Our study was specifically designed to investigate whether VPM neurons show direction selectivity when exposed to a multiwhisker stimulus generating an apparent motion across the whiskerpad. 
The thalamus is the last subcortical sensory station in the vibrissal pathway. However, VPM activity cannot be directly interpreted as the result of solely subcortical mechanisms. Indeed, the VPM is subject to dense excitatory feedback projections from upper layer VI of the primary somatosensory cortex (S1), with collaterals to the nRt (Bourassa et al., 1995; Land et al., 1995; Rouiller and Welker, 2000), so that both excitatory and inhibitory influences are exerted (Landisman and Connors, 2007; Lam and Sherman, 2010). To further investigate whether VPM response properties to apparent motion at the scale of the whiskerpad are generated subcortically or mediated by the corticothalamic feedback, we explored the selectivity of VPM neurons before and during inactivation of the barrel cortex.

\section{Materials and Methods}

\section{Animal preparation}

Twenty-four adult male Wistar rats that weighed 250-350 g and were obtained from the Animal Breeding Unit of our Institute were used for these experiments. Maintenance, manipulations, and surgery were performed in conformity with French (JO 87-848) and European legislation (86/609/CEE) on animal experimentation. After an initial induction of anesthesia with isoflurane ( $3 \%$ for $4 \mathrm{~min}$ ), the animals received an injection of urethane $(1.5 \mathrm{~g} / \mathrm{kg}$, i.p.) and an injection of atropine methyl nitrate $(0.3 \mathrm{mg} / \mathrm{kg}, \mathrm{i} . \mathrm{m}$.) to reduce secretions in the respiratory path. Supplemental doses of urethane $(0.15 \mathrm{~g} / \mathrm{kg}$, i.p.) were administered whenever necessary throughout the experiment to maintain an adequate level of anesthesia, indicated by the absence of eye blink reflex, response to hindpaw pinch, and vibrissae movement. The respiration was monitored throughout the experiment by means of a piezoelectric sensor placed between the chest and the platform on which the animal rested, and the electrocardiogram was recorded through two subcutaneous electrodes. Sudden increases of breathing rate or cardiac frequency prompted examination and correction of the anesthesia level by the experimenter. Additionally, the spectral content of the electroencephalographic activity was visually examined throughout the experiment to obtain recordings from Stage III-3 (Friedberg et al., 1999).

Body temperature was maintained at $37^{\circ} \mathrm{C}$ with a thermostatically controlled heating pad.

After the animal was mounted in a stereotaxic frame, a small craniotomy was drilled over the thalamic VPM (2-4 mm posterior, 2-4 mm lateral from bregma; Paxinos and Watson, 2007). For experiments with cortical inactivation, the craniotomy extended over the posteromedial barrel subfield ( $0-4 \mathrm{~mm}$ posterior, $4-7$ lateral from bregma; Chapin and Lin, 1984). The skull was cemented to a metal bar rigidly fixed to the stereotaxic frame, and the right ear bar was removed to allow free access to the right vibrissae.

\section{Electrophysiological recording}

Neural activity was recorded extracellularly by a tungsten electrode (FHC; $2-10 \mathrm{M} \Omega$ at $1 \mathrm{kHz}$ ) that was lowered vertically in the VPM (Fig. $1 A)$ using an electronically controlled microdrive (Luigs \& Neumann). For experiments with cortical inactivation, a second electrode was lowered in the barrel cortex perpendicularly to the surface. Signals were amplified (gain 5000) and filtered for spike activity (0.3-3 kHz; CyberAmp). For each recording site, 1-3 single units were isolated using a template-matching spike sorter (Alpha Omega). The shape of action potentials was continuously inspected to ensure that the same neurons were recorded throughout the protocols. Multiunit data were collected in the cortex by amplitude sorting. At the end of recording at a given site, the electrode was advanced by at least $100 \mu \mathrm{m}$ before the next recording site to avoid reporting data from the same units. Spike time acquisition and data processing were performed with a custom-made software (Elphy; G. Sadoc, Unité de Neuroscience, Information et Complexité, Centre National de la Recherche Scientifique).

\section{Whisker stimulation}

We used a custom-built multiwhisker stimulator, called the Matrix (Jacob et al., 2010), to independently move the 24 most caudal whiskers on the right side of the rat snout. Details of the whisker stimulation protocols have been described previously (Jacob et al., 2008). Briefly, each whisker was cut to a length of $10 \mathrm{~mm}$ and inserted into a short plastic tube glued to a piezoelectric bender (Polytec-PI). Individual whisker deflections consisted of pulses from the rest position to a more caudal position and then back ( $10 \mathrm{~ms}$ rise time, $10 \mathrm{~ms}$ hold, $10 \mathrm{~ms}$ fall time RC-filtered; initial velocity $93 \% \mathrm{~s}$ applied $7 \mathrm{~mm}$ from the skin). Whisker $\mathrm{C} 2$, in the center of the Matrix, was controlled by a multidirectional piezoelectric bender (Noliac) and could thus be moved in all directions.

Once one or more single units were isolated at a recording site, we first assessed their receptive fields by applying sparse noise stimulation. Approximately 200 pseudo-random sequences of individual deflections of the 24 whiskers were presented at $20 \mathrm{~Hz}(1.2 \mathrm{~s}$ intersequence interval). The receptive field maps were constructed online by forward correlation (Le Cam et al., 2011). This allowed us to rapidly estimate whether some of the recorded neurons responded preferentially to whisker $\mathrm{C} 2$, which was important for the apparent motion stimuli (see below).

If this was the case, we then applied multiwhisker stimulations consisting of individual caudal deflections of every whisker and mimicking a front-edge motion across the pad (Fig. $1 B$ ). We tested eight directions of movement spanning $360^{\circ}$ (one direction tested every $500 \mathrm{~ms}$; 128 repetitions per direction). Because whisker $\mathrm{C} 2$ sits at the center of the Matrix, and because we restricted our study to neurons for which C2 was the functional principal whisker, there was no a priori bias for a particular direction in this protocol. In a subset of cells, we applied the same stimulation protocol restricted to C2 and its eight neighboring whiskers ("proximal" motion selectivity protocol).

Additionally, we tested the response of the neurons to individual deflections of whisker $\mathrm{C} 2$ alone in the same eight directions (local direction selectivity protocol; $64-128$ repetitions per direction).

\section{Cortical inactivation}

To suppress the influence of the corticothalamic feedback, we applied $\mathrm{MgSO}_{4}$ (4\% in a saline solution) on the cortical surface after dura removal. This was repeated every $5 \mathrm{~min}$ until cortical activity recorded in deep layers was silenced, on average $35 \mathrm{~min}$ after the first application. Saline was then applied every $5 \mathrm{~min}$ until cortical activity recovered or until the end of the experimental session. We did not record from additional thalamic neurons once the cortex had been inactivated.

\section{Histology}

At the end of each experiment, two to three small electrolytic lesions (30 pulses of $200 \mathrm{~ms}$ and $10 \mu \mathrm{A}$ delivered at $0.3 \mathrm{~s}$ intervals) were made at known depths in the VPM and, if applicable, in the cortex, $500 \mu \mathrm{m}$ apart. The animal was given a lethal dose of pentobarbital and perfused transcardially with phosphate buffer $(0.1 \mathrm{M}, \mathrm{pH} 7.4)$ followed by a fixative solution (4\% paraformaldehyde in $0.1 \mathrm{~m}$ phosphate buffer, $\mathrm{pH} 7.4$ ). Coronal or oblique sagittal sections $(80 \mu \mathrm{m})$ were cut through the VPM and the barrel field and stained with cytochrome oxidase.

\section{Data analysis}

Quantification of responses using the surprise measure. We used the Poisson surprise method to assess the significance of neuronal responses to a given stimulus (Legéndy and Salcman, 1985). This method has been validated in a previous report (Le Cam et al., 2011). For all protocols, the first two trials were excluded from the analysis to ensure that only responses in the steady-state regime were included. Peristimulus time histograms (PSTHs) were constructed from repeated instances of single or multiwhisker deflections. A baseline firing rate was measured in a specific control window for each protocol. For the sparse noise protocol, we used a $150 \mathrm{~ms}$ control window right after the blank stimuli (no whisker movement) that were interspersed with the whisker deflections in the pseudorandom sequences, thus taking into account in the baseline level any delayed change of activity due to the rapid succession of whisker deflections. For the global motion or the local direction selectivity protocols, we used a $100 \mathrm{~ms}$ control window just before each multiwhisker stimulation in one direction. The surprise measure was then defined as the negative logarithm of the probability of observing a particular spike count in a bin, assuming a Poisson distribution of the spike counts around the baseline spike count value. To quantify the significance of 
A

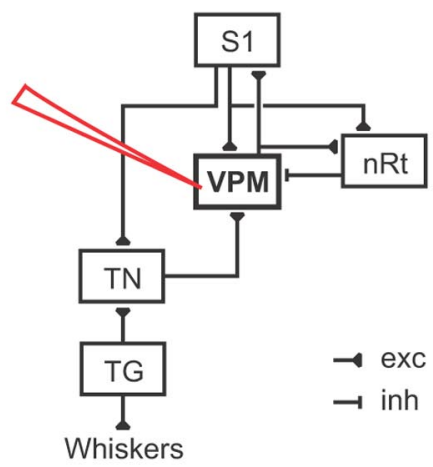

B

\section{Direction of global}

$0^{\circ}$

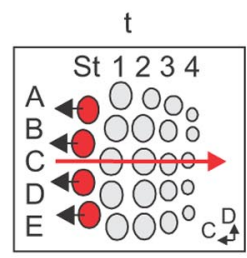

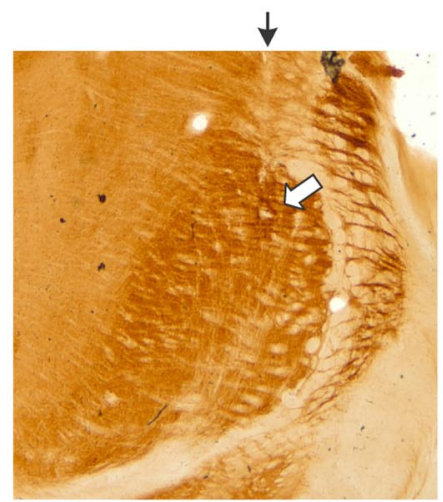

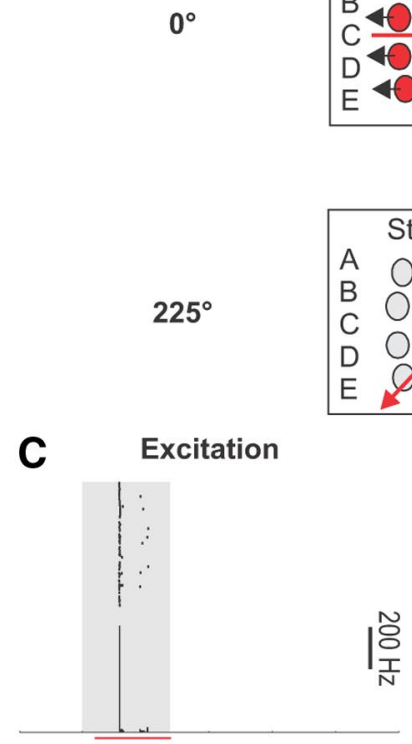

$\mathrm{t}$
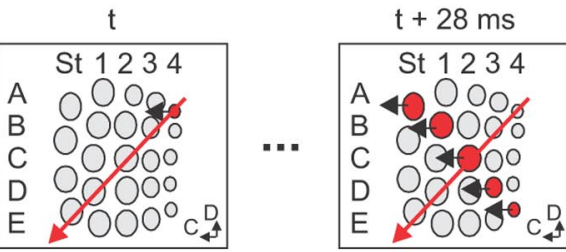

Excitation \& Suppression
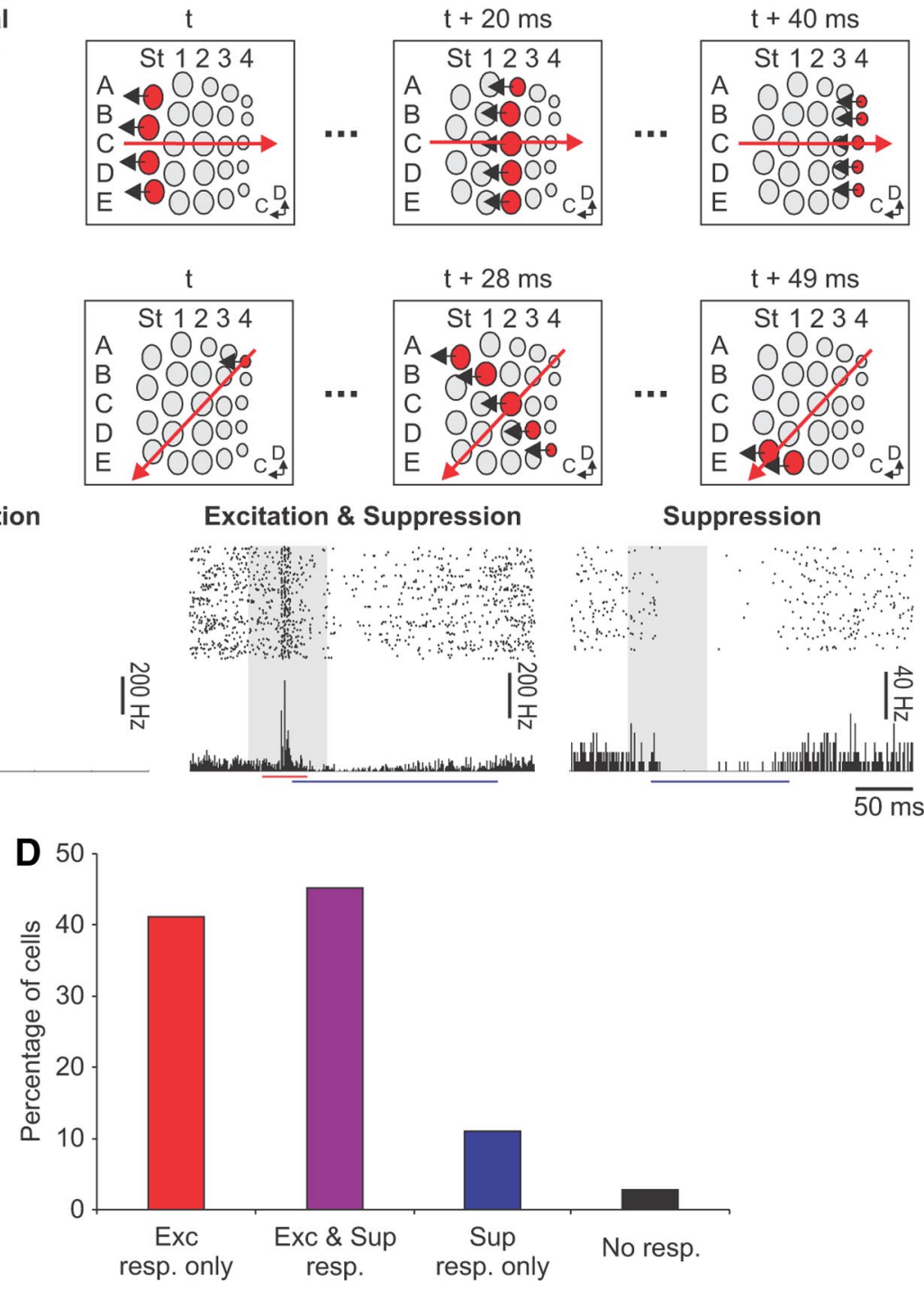

Figure 1. Response profiles of VPM neurons to multiwhisker stimuli. A, Left, Schematic diagram of the lemniscal pathway from the whiskers to the barrel cortex. Recordings were performed in the VPM of the thalamus. TG, Trigeminal ganglion; TN, brainstem trigeminal nuclei, including the PrV and the spV nuclei. Right, Histological verification that the recording sites were in the barreloid region of the VPM. Cytochrome oxidase staining was performed on coronal brain sections. The vertical track of the electrode (black arrow) and an electrolytic lesion (white arrow) in the core region of VPM barreloids are visible. $\boldsymbol{B}$, Details of the spatiotemporal pattern of stimulation for two directions of global motion $\left(\right.$ top, $0^{\circ}$; bottom, $225^{\circ}$ ) among the eight directions tested. Small black arrows indicate the local motion of each whisker, always in a caudal direction. Whiskers for which mechanical stimulation starts at the time indicated on top of each frame are colored in red. Only three frames are drawn for each direction. The large red arrows recapitulate the apparent global motion across the whiskerpad. The left column contains the four straddlers (St). Caudal (C) is left, rostral (R) right, dorsal (D) up, ventral (V) down. These conventions apply for all figures. C, Three examples of firing rate profiles in response to multiwhisker stimulation in one direction (left, $270^{\circ}$; middle and right, $0^{\circ}$ ) from three different neurons. On the top, each line of the raster plot represents the action potentials fired during one trial (126 trials in every example). On the bottom, PSTHs are obtained by summing the activity in $1 \mathrm{~ms}$ bins and normalizing by time. Gray shading indicates the duration of stimulus presentation. Colored lines below the histograms indicate windows of significant excitatory (red) and suppressive (blue) changes in firing rate (see Materials and Methods). D, Percentages of cells for which excitatory and suppressive components of response could be defined. All cells for which C 2 was defined as the functional principal whisker and which could be tested with the multiwhisker protocol are included $(n=73)$. exc, Excitatory; inh, inhibitory; sup, suppressive; resp, response; t, time. 
both sharp responses and temporally spread ones, this analysis was performed in parallel for 20 bin sizes ranging from 1 to $20 \mathrm{~ms}$. A threshold above which surprise values were considered significant was determined such that $<1 \%$ of control-window PSTHs across the whole population were found to be false positives, that is, to have at least one surprise value exceeding the threshold. Thresholds were computed independently for the 20 bin sizes and for activity values above and below baseline levels (respectively, excitation and suppression effects). This enabled us to define, for each cell and protocol, a putative excitation window as the union of all bins exhibiting a significant "excitatory" surprise value, and similarly, a putative suppression window. Note that because the analysis was tailored to identify changes on multiple time scales from 1 to $20 \mathrm{~ms}$, the windows of putative changes of activity could be larger than the response phases themselves.

Response amplitudes in number of spikes per stimulus ( $\mathrm{sp} / \mathrm{stim})$ were obtained by integrating the firing rate relative to the baseline level in those windows. Note that integration was performed on the same window of analysis for all PSTHs of a given protocol ( 8 for the 8 directions in the multiwhisker stimulation protocol, 24 for the 24 whiskers in the receptive field protocol), whether each particular PSTH exhibited significant changes in activity or not. The window of integration could not extend outside of $0-150 \mathrm{~ms}$ for the receptive field estimation, and outside of 0-90 and 0-250 ms, respectively, for the excitatory and suppressive components in both the multiwhisker global motion protocol and the local direction selectivity protocol. These limits were defined from the population statistics of response profiles to focus on the first excitatory and suppressive components of the response, without including the rebound excitatory phase that was occasionally observed. By convention, response amplitudes for the suppression components were assigned positive values.

Receptive field estimate. For the sparse noise protocol, a response latency after a given whisker deflection was defined as the end time of the first bin in the PSTH for which a surprise value was significant (for any bin size). Latencies were computed separately for excitatory and suppressive phases of response. The receptive field of a neuron was defined as the set of whiskers for which a significant response was obtained with a latency in the window of 5-55 ms. Responses could have excitatory and/or suppressive phases. We calculated response amplitudes for all whiskers as indicated above, leading to two response maps, one for the excitatory and one for the suppressive component, on which the whiskers eliciting significant responses were delineated. The functional principal whisker of the neuron was defined as the whisker for which the latency of the excitatory phase of the response was the shortest. When responses to several whiskers had equal latencies, we used the latencies of the multiunit activity recorded simultaneously by the electrode (unsorted waveforms). In case the multiunit activity also yielded equal latencies, we selected the whisker eliciting the largest single-unit response magnitude. In a few cases ( $n=7$ in the sparse noise protocol) with no short-latency excitatory response but with a prominent suppressive component, the functional principal whisker was defined as the whisker eliciting the largest suppression of activity.

To determine a main axis of the receptive field, we calculated a center of mass separately for suppression and excitation response maps using the whiskers eliciting significant responses weighted by their response amplitudes. An asymmetry vector was defined, linking the functional principal whisker $(\mathrm{C} 2)$ to the center of mass.

Quantification of the direction selectivity protocol. For the three stimulation protocols testing the selectivity to the direction of motion (global motion, proximal motion, local C2 motion), response magnitudes were calculated as described above, and the tuning curves for the excitatory and suppressive components were represented as polar plots. The eight vectors connecting the center to the eight points of the tuning curve were added and the resulting sum vector displayed as a thick line on the polar plot. The direction of this vector, i.e., the circular mean of the tuning curve, was taken as the preferred direction whenever the direction selectivity was found to be significant (Rayleigh test on the spike counts for the eight directions, $p<$ 0.05) (Fisher, 1996). The magnitude of the direction selectivity was quantified by a direction index (DI) equal to the ratio of the sum vector length over the sum of the individual tuning curve values (Swindale, 1998).

During the experiments with cortical inactivation, the multiwhisker and sparse noise stimulation protocols were continuously alternated throughout the inactivation and recovery periods; thus several tuning curves could be acquired during cortical inactivation for a given cell. For the population analysis, we systematically selected the first multiwhisker stimulation protocol for which cortical spontaneous and evoked activity levels had dropped below $95 \%$ of initial values.

Statistical analysis. Results are displayed as mean \pm SEM except where explicitly stated. For analysis of the relationship between two circular (angular) variables, we used a statistical correlation test based on the method by Fisher (1996).

\section{Results}

We recorded the spiking activity of single units in the left VPM nucleus of the rat thalamus during whisker stimulation. Off-line analysis confirmed that $\mathrm{C} 2$ was the functional principal whisker (see Materials and Methods) for 73 of these neurons, which were considered for subsequent analysis. We verified that the recording sites were in the barreloid region of the thalamus using cytochrome oxidase staining (Fig. $1 A$ ).

\section{VPM neurons respond to multiwhisker stimulation with different response profiles}

Using a Matrix stimulator (Jacob et al., 2010), we tested the responses of VPM neurons to patterned stimulation of 24 whiskers on the contralateral pad. The movement of every whisker was a calibrated $114 \mu \mathrm{m}, 30 \mathrm{~ms}$ caudal deflection; however, the spatiotemporal order of individual deflections was such that for each multiwhisker presentation, an apparent global motion of the whiskers occurred in one of eight possible directions (Fig. $1 B$, see details for two directions).

We observed three different types of response profiles evoked by the multiwhisker stimuli, as illustrated in Figure $1 C$. The first class of responses was characterized by a pure increase in firing rate, often tightly locked to the stimulus presentation and of short duration. For example, the first cell of Figure $1 C$ emitted one action potential at a precise time during stimulus presentation on most trials, leading to a $1 \mathrm{~ms}$ wide peak of activity in the response profile. The second class of responses consisted of biphasic profiles with an initial peak followed by a decrease of activity below the baseline level (Fig. 1C, middle). In the third class of responses, the activity dropped below the baseline level and later recovered (Fig. 1C, right). As is typical of sensory responses, periods of suppression of activity exhibited slower kinetics than periods of increases in firing rate. We quantified both the excitatory and suppressive components of responses using a surprise-based method (see Materials and Methods) (Le Cam et al., 2011), which identifies windows of significant changes in firing rate relative to the spontaneous activity baseline level.

For each neuron, we looked at which response components could be detected after the multiwhisker presentation for at least one of the eight directions of apparent motion (Fig. 1D). Thirty cells of the $73 \mathrm{C} 2$-centered cells that were tested were characterized by the presence of an excitatory response component with no suppression of activity. In addition, 33 cells could exhibit both excitatory and suppressive response components, so that a total of $86 \%$ of the neurons showed an increase of firing rate in at least one PSTH. Another group of cells $(n=8)$ displayed only periods of suppression of activity. In addition, two cells did not show any significant response to the multiwhisker stimuli, even though we had determined previously that $\mathrm{C} 2$ was their functional principal whisker using the sparse noise stimulation protocol. These results confirm that evoked responses most often involved increases in firing rate, and that periods of suppression of activity could be elicited in more than half of the neurons. 
A1
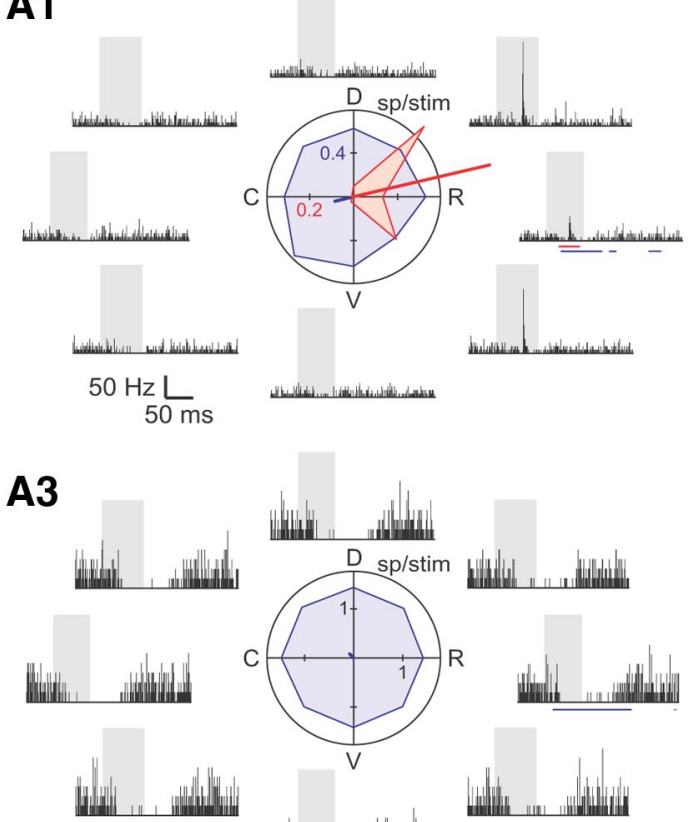

$20 \mathrm{~Hz} L$

$50 \mathrm{~ms}$

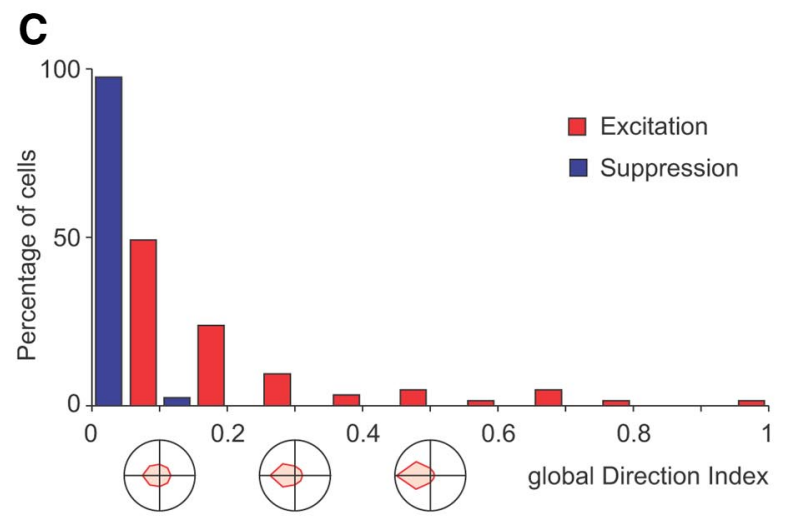

A2

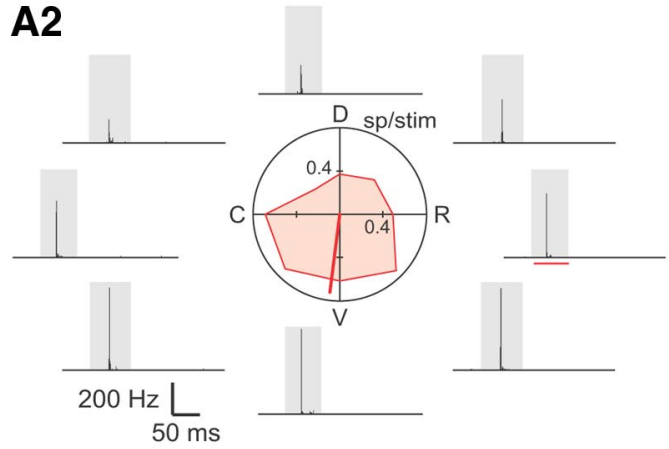

B

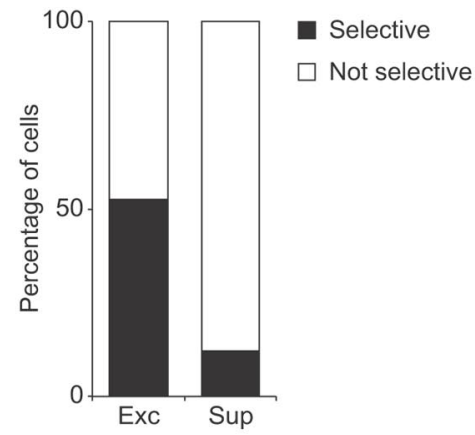

D

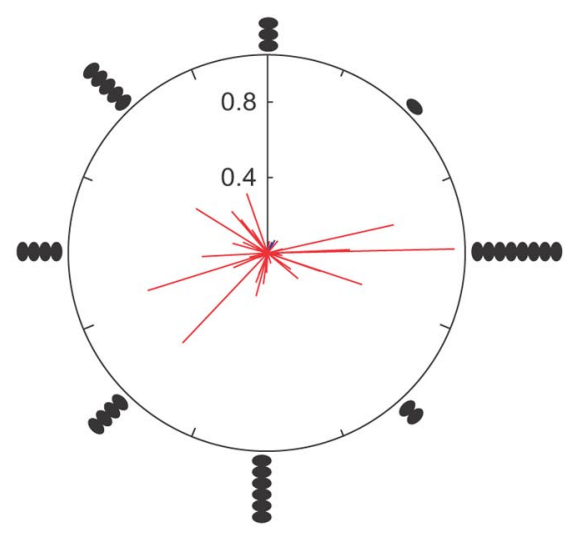

Figure 2. Selectivity of VPM responses for the direction of motion of the multiwhisker stimulation. A1-A3, PSTHs of response and tuning curves of excitatory and suppressive response components for three VPM neurons. Firing rate histograms were constructed as described in Figure 1 and arranged around the central circle by angle of global motion. Gray shading indicates the period of stimulus presentation (horizontal and vertical, $0-70 \mathrm{~ms}$; oblique, $0-87 \mathrm{~ms}$ ). A unique excitatory (respectively suppressive) window of firing rate changes was determined by combining the eight windows obtained for the eight directions (see Materials and Methods). These windows, indicated as red and blue lines under the PSTH for $0^{\circ}$, were used to calculate the response magnitudes appearing in the central polar plots (excitatory component, red tuning curve; suppressive component, blue tuning curve). The thick red (respectively, blue) line originating from the center is the vector sum of the eight excitatory (respectively suppressive) responses in the polar plot, and its length is used to calculate the DI by dividing it by the sum of response magnitudes. When a significant selectivity exists, as for the excitatory response in the first two examples, the angle of the vector sum gives the preferred direction. The excitation and suppression polar plots in $\boldsymbol{A} \mathbf{1}$ are displayed with different scales indicated by the red (excitatory) and blue (suppressive) numbers. Conventions apply to all other figures. $\boldsymbol{B}$, Percentages of neurons with a significant selectivity for global motion (black bars). The excitatory and inhibitory response components were analyzed separately (excitatory, $n=63$; suppressive, $n=41$ of 73 neurons). C, Distributions of the global Dls for the excitatory (red, $n=63$ ) and inhibitory (blue, $n=41$ ) response components. D, Excitation (red, $n=33$ ) and suppression (blue, $n=5$ ) direction vectors for all cases with a significant selectivity. The vector length is the DI and the vector angle is the preferred direction for the cell. The histogram around the plot shows the number of direction vectors in $45^{\circ}$ intervals. There was no indication of anisotropy for a particular angular direction or orientation (Rayleigh test on $0-360^{\circ}$ and $0-180^{\circ}, p>0.4$ in each case).

Average response magnitudes were similar to those after stimulation of $\mathrm{C} 2$ only at the same rate, both for excitation (mean \pm SD: multiwhisker $0.8 \pm 0.7 \mathrm{sp} / \mathrm{stim}$ vs single-whisker response $0.8 \pm 0.8 \mathrm{sp} / \mathrm{stim}$; Wilcoxon paired sample test, $p=0.74, n=58$ ) and for suppression (multiwhisker $0.9 \pm 0.9 \mathrm{sp} / \mathrm{stim}$ vs singlewhisker response $0.8 \pm 0.8 \mathrm{sp} / \mathrm{stim} ; p=0.54, n=28$ ).

\section{VPM neurons are selective to emergent properties of multiwhisker stimuli}

In some neurons, the response to the multiwhisker stimuli depended heavily on the direction of apparent motion. Figure $2 \mathrm{Al}$ shows the responses of a neuron which increased its firing rate phasically during the stimulus presentation, but exclusively for three directions $\left(-45^{\circ}, 0^{\circ}\right.$, and $\left.45^{\circ}\right)$. The activity of this neuron also displayed a suppressive component, which, by contrast to the excitatory component, was present and relatively constant for all directions of global motion. We integrated spike counts above and below the spontaneous activity level and constructed polar plots of response magnitude as a function of the angle of global motion of the multiwhisker stimulus. Thus, the tuning curve of the neuron in Figure $2 \mathrm{Al}$ for the excitatory component of the response occupies the right quadrant of the plot around $0^{\circ}$, 
A
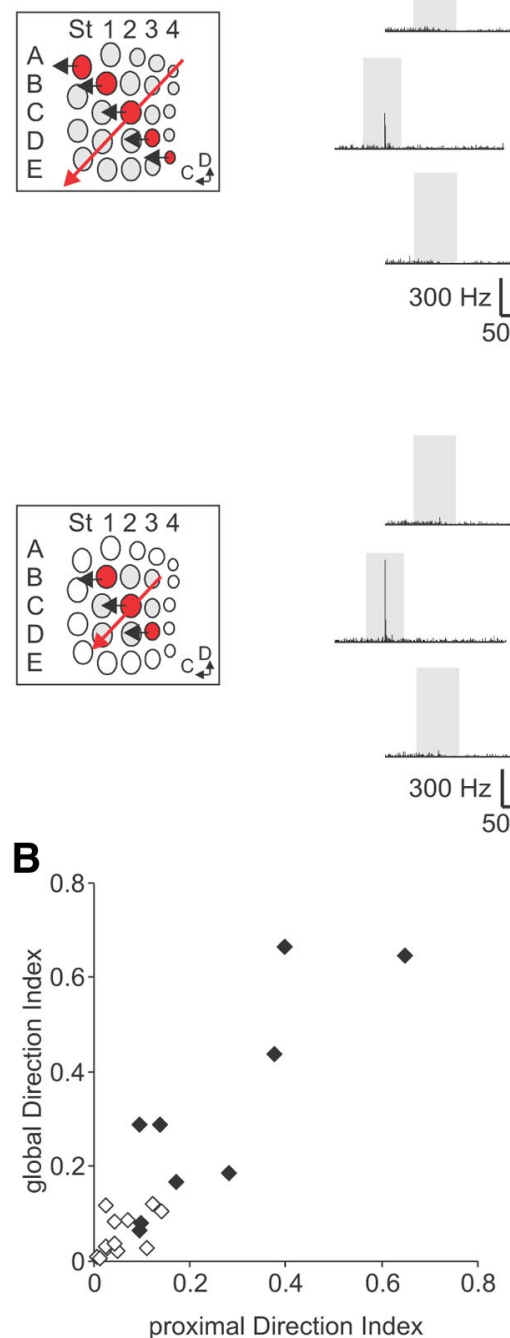

C
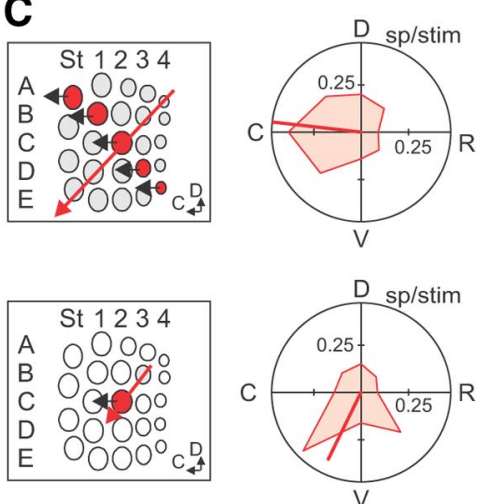
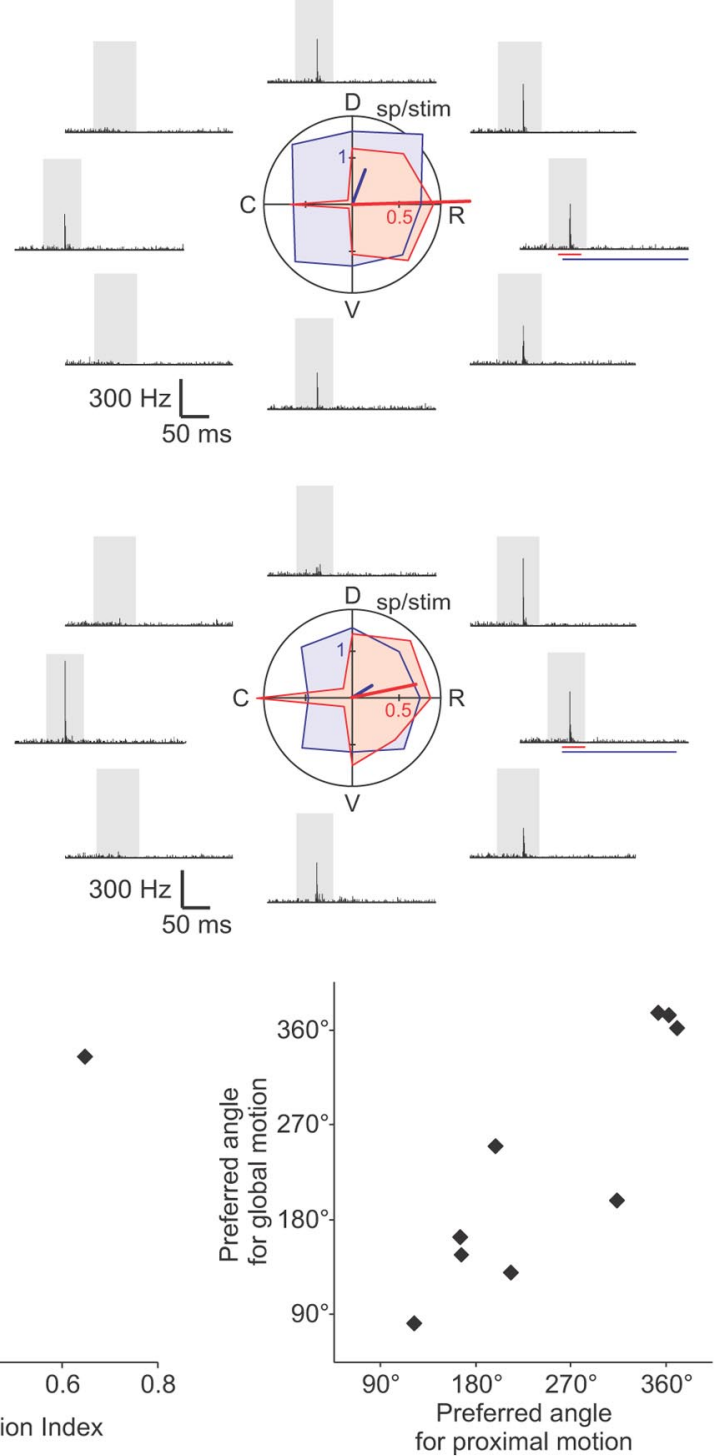

D

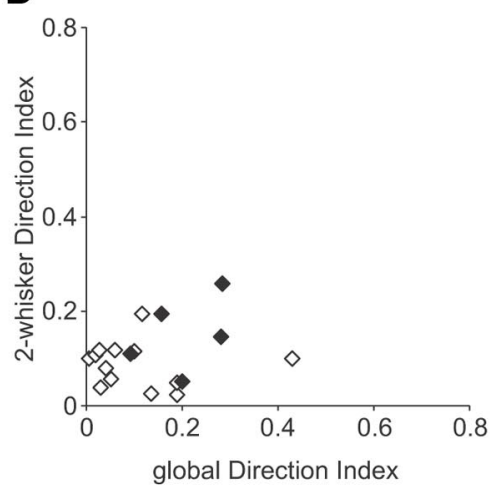

Figure 3. Direction selectivity to apparent motion across nine or two whiskers. $\boldsymbol{A}$, PSTHs of response and tuning curves of excitatory and suppressive response components for the global (top) and proximal (bottom) motion stimuli for the same VPM neuron. Left (inset), Patterns of stimulation for the $225^{\circ}$ angle for the two protocols. In the proximal configuration, only the central whisker $\mathrm{C} 2$ and the eight adjacent surrounding whiskers (red/gray) were stimulated, all other whiskers were at rest (white); all other parameters were unchanged. $\boldsymbol{B}$, Left, Scatter plot of the Dls in the proximal (horizontal axis) and global (vertical axis) motion protocols for all cells exhibiting an excitatory component of response in both $(n=22)$. Black diamonds indicate cells for which both protocols resulted in a significant selectivity for direction $(n=9)$. Right, Scatter plot of preferred angles for this last subset of cells. There was a significant correlation of the DIs $\left(r^{2}=0.8, p=1.10^{-8}\right)$ and of the preferred angles $(\rho=0.4, p=0.018)$ in the two protocols. C, Tuning curves of response for the global (top) and two-whisker (bottom) motion stimuli for one VPM neuron. Left

whereas the suppressive component tuning curve is close to isotropic. Circular quantification and statistics (see Materials and Methods) confirmed a high and significant angular selectivity of the excitatory component of the response (DI $=$ 0.66; Rayleigh test, $p<0.0001$ ) but not of the suppressive component $(\mathrm{DI}=0.03$; Rayleigh test, $p=0.47)$. We conclude that the response of this neuron depended on the apparent direction of the multiwhisker stimulus. Under a linear sum scenario, because the deflections of individual whiskers were always identical, the tuning curve should be isotropic. Thus, this functional property necessarily emerges from nonlinear processing of information across the whiskerpad. Figure $2 A 2$ shows the example of a second neuron for which prominent excitatory components were visible for all directions, but were larger for ventral directions, resulting in a highly significant selectivity (DI $=0.17$; Rayleigh test, $p<0.0001)$. On the third example in Figure $2 A 3$, the neuron displays a period of suppression of activity with no angle selectivity.

Overall, the excitatory response component, when it was present, displayed a selectivity for the direction of the global stimulus in $52 \%$ of the cases (Fig. $2 B ; n=$ $33 / 63$ cases; Rayleigh test, $p<0.05)$. In contrast, only $12 \%$ of the cells (5/41 cells) displayed a direction selectivity of the suppressive response component (Rayleigh test, $p<0.05)$. Direction selectivity for the suppressive phases of response was never observed in suppressive-only cells (Fig. $2 A 3$, as the cell displayed).

The histograms of Figure $2 C$ show the corresponding distributions of indices of selectivity. Suppression DIs (in blue) are almost all grouped in the first bin from 0 to 0.1 , confirming that decreases in activity varied little with the angle of stimulation. DIs for excitation (in red) could take higher values, in parallel to the larger percentage of selective cases.

All individual whisker deflections were applied with a fixed local direction $\left(180^{\circ}\right)$.

\footnotetext{
(inset), Patterns of stimulation for the $225^{\circ}$ angle for the two protocols. In the two-whisker configuration, only the central whisker $\mathrm{C} 2$ and the closest adjacent whisker just preceding it (red/gray) in the sequence were stimulated, all other whiskers were at rest (white). D, Scatter plot of the Dls in the global (horizontal axis) and 2-whisker (vertical axis) motion protocols for all cells exhibiting an excitatory component of response in both $(n=18)$. Black diamonds indicate cells for which both protocols resulted in a significant selectivity for direction $(n=5)$. There was no significant correlation of the $\operatorname{Dls}\left(r^{2}=0.05, p=0.36\right)$ in the two protocols.
} 
We thus questioned whether the global motion preferred directions of VPM cells exhibited a bias toward a particular angle or not. The distribution of preferred angles of the excitatory component of the response showed no significant anisotropy across the population (Fig. $2 D$; Rayleigh test, $p=0.76, n=33$ ).

\section{The global motion selectivity of VPM neurons is generated mostly by the proximal whiskers}

The multiwhisker stimulus encompassed whisker C2 and 23 neighboring whiskers, thus involving most of the large macrovibrissae on one side of the snout. Given that VPM receptive fields are usually small, consisting of only a few vibrissae (Shosaku, 1985; Chiaia et al., 1991; Brecht and Sakmann, 2002), we hypothesized that the global motion selectivity might require only the stimulation of $\mathrm{C} 2$ and its eight adjacent whiskers, but not of more peripheral whiskers on the second ring around C2. Thus, we applied the same multiwhisker protocol but restricted to $\mathrm{C} 2$ and the eight adjacent whiskers, and compared the activity to that obtained by the full (global) multiwhisker protocol. Figure $3 \mathrm{~A}$ shows the PSTHs for a VPM neuron in the two protocols. Both were characterized by sharp excitatory peaks followed by a prolonged suppression of activity for all directions of apparent motion except two $\left(135^{\circ}\right.$ and $\left.225^{\circ}\right)$. Quantification of response magnitudes confirmed similar excitation and suppression tuning curves in the two protocols. This comparison was attempted on 31 C2-centered cells, of which 22 had a significant excitatory component of response in the two protocols, allowing calculation of direction selectivity indices. The global and proximal DIs were significantly correlated (Fig. 3B, left; Pearson's coefficient of determination, $\left.r^{2}=0.8, p=1.10^{-8}, n=22\right)$, and so were the preferred angles (Fig. $3 B$, right; angular-angular correlation test for significantly selective cases, $\rho=0.4, p=0.018, n=9$ ). The similarity of the responses to the two protocols suggests that indeed, the mechanisms of global motion selectivity for VPM neurons mostly involve the proximal periphery around $\mathrm{C} 2$.

These results raised the question of whether stimulating C2 and only one neighboring whisker at a time would suffice to generate the observed tuning curve for apparent motion. We thus applied a 2-whisker apparent motion protocol on 19 VPM neurons and compared responses to those obtained with the full 24-whisker protocol on the same cells. Figure $3 C$ shows the response values for a neuron which exhibited significant direction selectivity in both conditions; the tuning curves were different and the direction vectors pointed toward angles almost $90^{\circ}$ apart. At the population level, the indices of selectivity were unrelated (Fig. 3D; Pearson's coefficient of determination, $r^{2}=0.05, p=$ $0.36, n=18$ ), and when both tuning curves were significantly anisotropic, the preferred direction angles were not correlated either (angular-angular correlation test for significantly selective cases, $\rho=0.26, p=0.18, n=5$ ).

In this 2-whisker protocol, the set of whiskers stimulated is unique for each of the eight directions, so that the linear prediction of the response from the responses to individual deflections is not isotropic anymore as in the 24- or 9-whisker versions. Instead, it depends directly on the spatial characteristics of the suprathreshold receptive field. Nonetheless, the discrepancy between the tuning curves for the global motion and the twowhisker stimuli indicates that the global motion selectivity cannot be explained only by interactions between $\mathrm{C} 2$ and the immediate neighbor preceding it in each sequence. We conclude that the global motion selectivity of VPM neurons emerges from integration of information across C2 and the eight whiskers surrounding it.
Global motion selectivity was observed for VPM neurons with diverse suprathreshold receptive fields

Sensory physiology seeks to understand how responses to complex stimuli are generated, thus building a comprehensive model of sensory neurons. For the vibrissal system, a natural attempt is to explain neuronal responses to multiwhisker stimuli from responses to individual deflections of each whisker. Here, we explored whether the angular selectivity for global motion of VPM neurons could be related to single-whisker response properties. We estimated the receptive field of each neuron by quantifying responses to a sparse noise protocol, consisting of nonoverlapping short deflections applied in a pseudo-random order to each of the 24 whiskers. As for the multiwhisker stimulation protocol, significant periods of excitation or suppression of activity were identified on the firing rate profiles (Fig. $4 A 1, B 1$ ), and spike counts were integrated in these windows for each whisker. This analysis yielded two response maps, one for the excitatory and one for the suppressive component, on which we delineated the subsets of whiskers for which the response component was significantly different from baseline (Fig. 4A2,B2). VPM neurons could exhibit either monovibrissal (Fig. $4 A$, significant response to only one whisker; $n=45$ ) or multivibrissal (Fig. $4 B$, significant responses to more than one whisker; $n=21$ ) excitatory response maps. Suppression of activity, when it was present, was generally observed for several whiskers (two or more whiskers: $n=31$; one whisker only: $n=5$; no suppression: $n=37$ ) (Fig. 4C). Seven neurons showed exclusively suppression of activity following stimulation, with no excitatory response component.

Despite this diversity in receptive field properties, we found no relationship between the extent or structure of the receptive field and the angular selectivity measured with the multiwhisker stimulation. For example, both cells in Figure 4 were highly selective to the direction of global motion (Fig. 4A3,B3), while their receptive fields differed widely in spatial extent of the excitatory and suppressive components. At the population level, there was no difference in the distributions of DIs between monovibrissal and multivibrissal neurons, whether we considered the excitatory response map alone or a combined map for the two response components (Mann-Whitney test, $p>0.3$ in both cases). Likewise, the selectivity for global motion of VPM neurons was similar whether their receptive fields included a suppressive component or not (Mann-Whitney test, $p=0.2$ ). DI values were not correlated with the number of whiskers in the receptive field (Pearson's coefficient of determination, $r^{2}=$ $0.005, p=0.56$; Fig. $4 D$ ).

Nonetheless, we reasoned that for multivibrissal neurons, the spatial structure of the receptive field might shape multiwhisker responses so that their angular selectivity would reflect a geometrical property of the receptive field. To investigate this issue, we calculated for each response map an asymmetry vector linking the functional principal whisker $(\mathrm{C} 2)$ to the center of mass of the map (see Materials and Methods). There was no correlation between the asymmetry magnitude and the selectivity index, nor between the asymmetry angle and the multiwhisker stimulation preferred angle, both when excitatory and suppressive components were considered (Pearson's coefficient of determination, $r^{2}<0.05, p>0.2$ for comparisons between linear variables; angular-angular correlation test, $|\rho| \leq 0.1, p>0.7$ for comparisons between angles) (Fig. $4 E$; scatter plot with the excitatory response components). Thus, as for cortical neurons (Jacob et al., 2008), we found that the emergence of a selectivity for a specific angle of global motion across the whiskerpad could occur whatever the extent or structure of the receptive field of the neuron. 

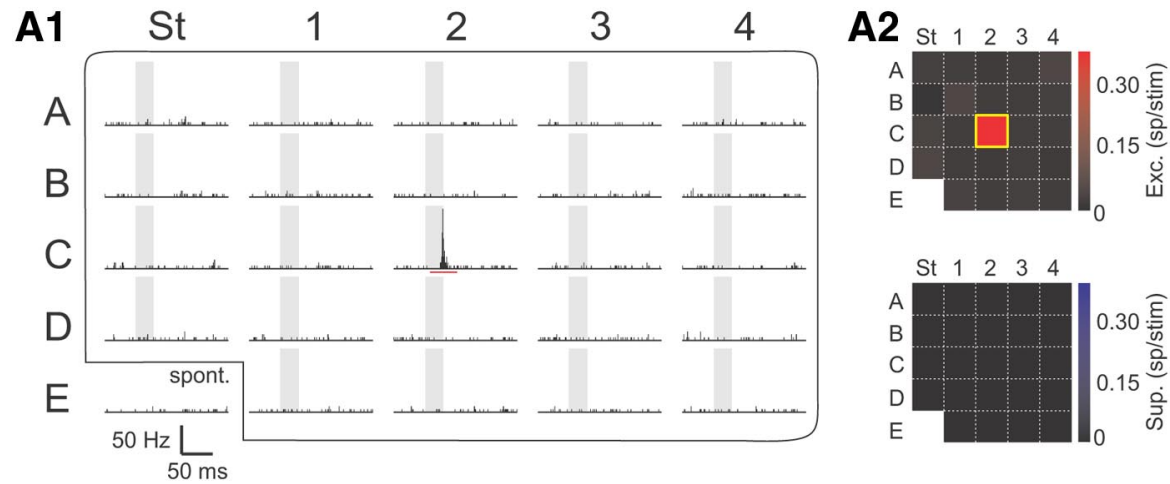

A3
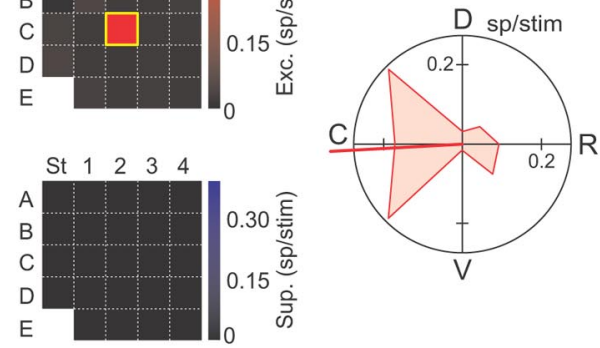

B1

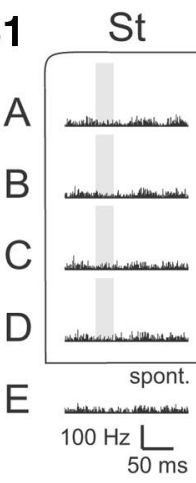

C

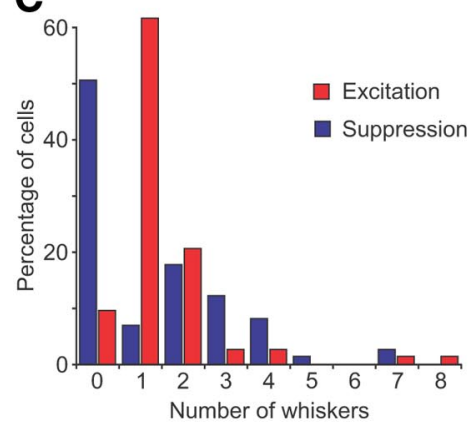

3

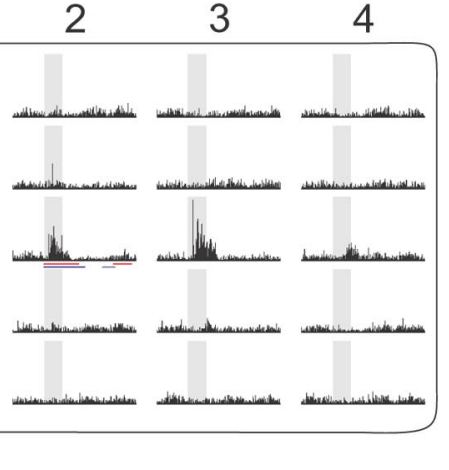

D

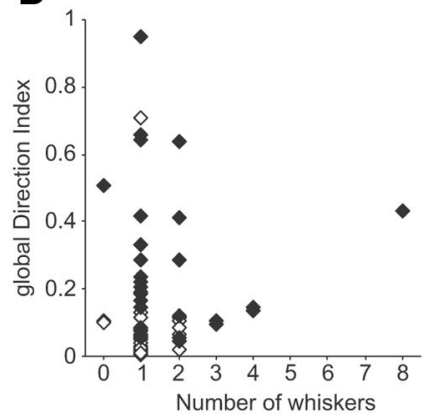

B2
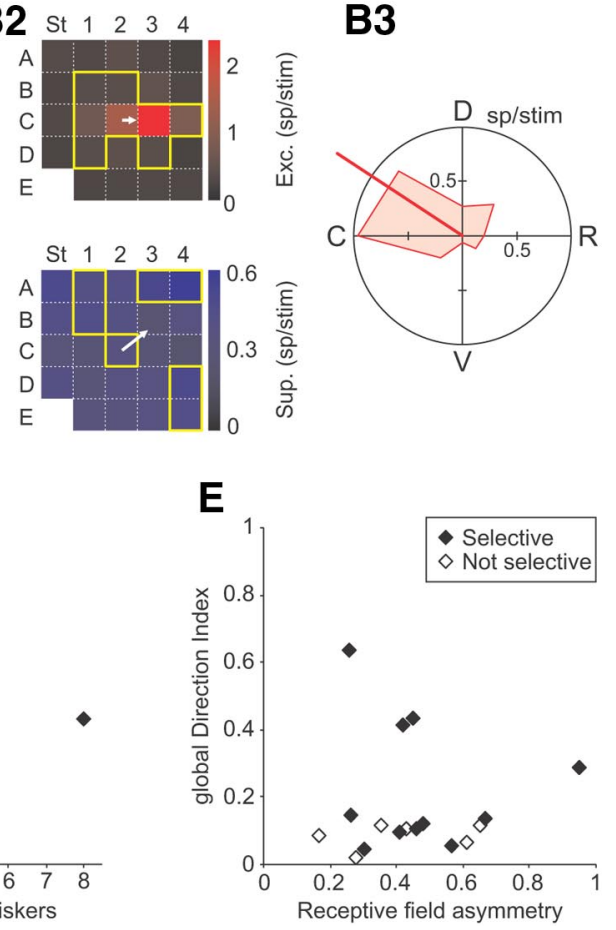

Figure 4. Receptive field response maps of VPM neurons and their relationship to the direction selectivity for global motion. $A 1, B 1$, PSTHs of response to single-whisker caudal deflections in the sparse noise protocol for two VPM neurons. Gray shading indicates stimulus presentation ( $30 \mathrm{~ms}$ ). Blanks in the stimulation sequence were used to assess spontaneous activity levels (spont., bottom left) in addition to the 24 whisker-related PSTHs. The lines below the C2 PSTH indicate the windows on which spike counts were integrated above (red) and below (blue) the spontaneous activity level to obtain response magnitudes; these windows were the same for all PSTHs of a given cell (see Materials and Methods). A2, B2, Excitatory (top) and suppressive (bottom) response maps for these two neurons. The yellow lines delineate whiskers for which the PSTH surprise analysis determined a significant response compared with baseline. White arrows show the asymmetry vectors (see Materials and Methods). A3, B3, Polar plots of responses to the multiwhisker global motion protocol for the same two neurons. There was no suppression of activity. Both cells displayed a high selectivity for the direction of global motion $(\mathrm{DI}=0.33$ and $0.43, p<0.001)$. C, Distributions of the number of whiskers eliciting a significant excitatory (red) and inhibitory (blue) response component $(n=73)$. D, Global DI of the excitatory response component as a function of the total number of whiskers with a significant excitatory response ( $n=63$ cells for which the DI could be calculated). There was no correlation between the two variables $\left(r^{2}=0.0055, p=0.56\right)$. Black diamonds indicate cells with a significant direction selectivity $(n=33)$. $E$, Global Dl of the excitatory response component as a function of the asymmetry in the excitatory response map ( $n=21$ cells for which an asymmetry could be calculated). There was no correlation between the two variables $\left(r^{2}=0.0002, p=0.96\right)$. Black diamonds indicate cells with a significant direction selectivity $(n=11)$.

These results suggest that global motion selectivity either arises from subthreshold integration mechanisms or is already present in the inputs to VPM.

\section{The global motion selectivity is distinct from the direction selectivity for local motion of whisker C2}

A spatial gradient for the preferred angle of principal whisker deflection has been described in VPM barreloids (Timofeeva et al., 2003) - the VPM equivalent to the pinwheel maps for local angular selectivity in the cortical barrels (Andermann and Moore, 2006; Kremer et al., 2011). A parallel organization for the global motion selectivity could exist as well, and translate into a link between these selectivities, in particular if they are generated by common mechanisms. Thus, we examined whether the selectivity for global motion of VPM neurons was related to their selectivity for the angle of deflection of the principal whisker.

To investigate this hypothesis, whisker C2 was deflected pseudo-randomly in eight possible directions ( $n=68$ neurons). In Figure $5 A$, the first example displays a neuron for which strong phasic excitatory responses were evoked by deflections in dorsal directions, and less in ventral directions. For this same neuron, the multiwhisker stimulation protocol revealed a strong selectivity to the global motion angle, but the two tuning curves appeared unrelated. On the second example, the neuron is selective to the direction of motion of $\mathrm{C} 2$ but not selective to the multiwhisker 
A
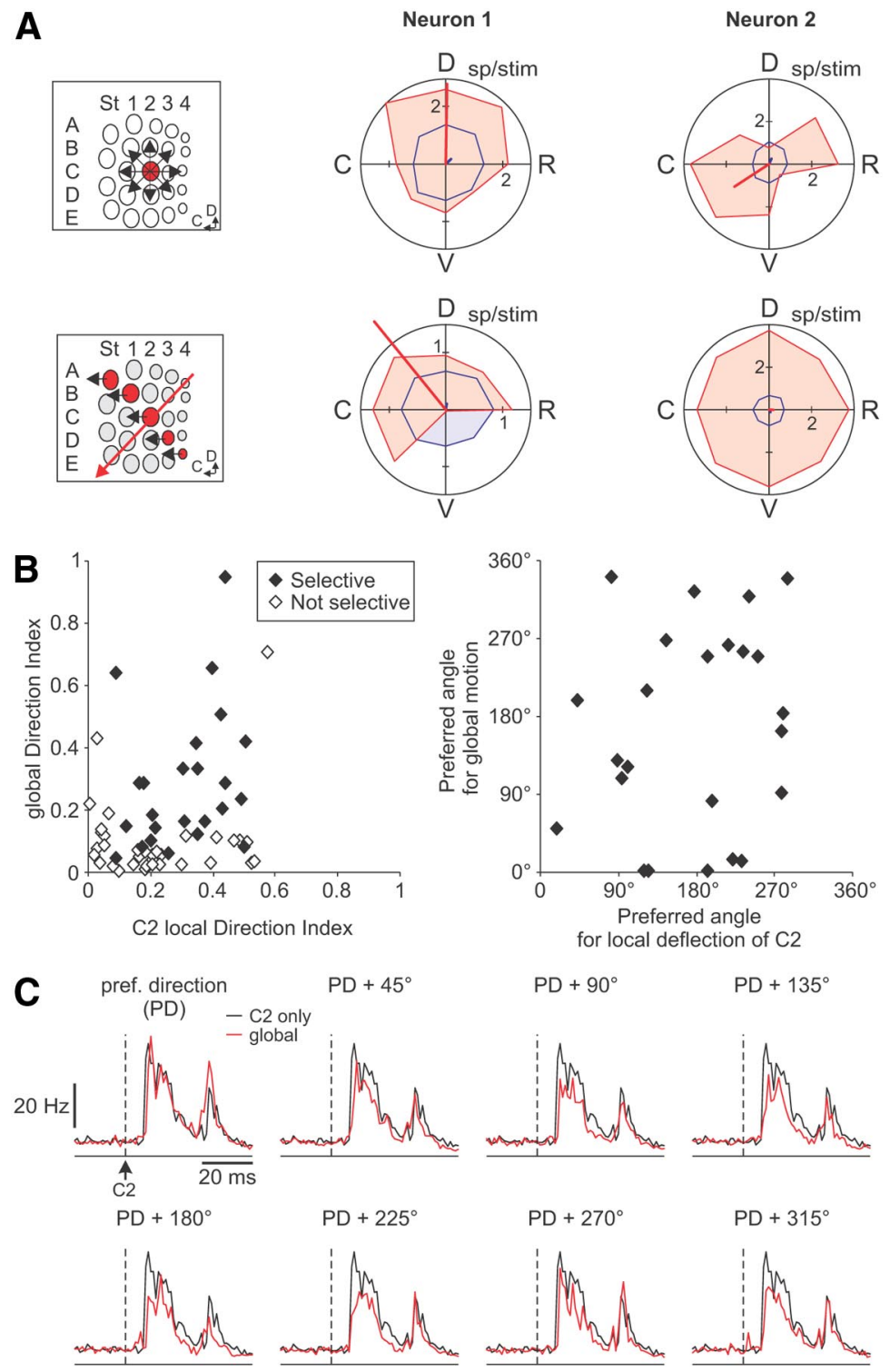

Figure 5. Comparison of direction selectivities for the local deflection of $\mathrm{C} 2$ and the global motion protocol. $\boldsymbol{A}$, Polar plot tuning curves of excitatory and suppressive response components for the local deflection of $C 2$ (top) and the global motion stimuli (bottom) for two VPM neurons. Left (insets), Schematic diagrams of the deflection of whisker $C 2$ in eight possible directions (1 per trial, all other whiskers were at rest) and the pattern of stimulation for the $225^{\circ}$ angle for the global motion protocol. $\boldsymbol{B}$, Left, Scatter plot of the excitation DIs in the local (horizontal axis) and global (vertical axis) motion protocols for all neurons exhibiting an excitatory component of response in both protocols ( $n=58$ among the 68 neurons tested). Black diamonds indicate neurons with a significant selectivity for direction in both protocols $(n=24)$. Right, Scatter plot of preferred angles for this last subset of neurons. There was no significant correlation of the DIs $\left(r^{2}=0.08, p=0.19\right)$ and of the preferred angles $(\rho=-0.05, p=0.89)$ in the two protocols. C, PSTHs of response to C 2 caudal deflections (black) and global motion stimuli (red) averaged across all neurons tested in the two conditions $(n=68)$, for the preferred direction and the seven other directions of global motion. All PSTHs are aligned by the onset of the 22 stimulus (dashed line). Global motion stimuli extend from -20 to $50 \mathrm{~ms}$ for vertical and horizontal directions and from -28 to $58 \mathrm{~ms}$ for oblique directions.

apparent motion angle. Overall, 24 cells showed significant direction selectivity for both the multiwhisker and the $\mathrm{C} 2$ stimulation protocols. Their direction selectivity indices were not correlated (Fig. 5B, left; Pearson's coefficient of determination, $r^{2}=0.08$, $p=0.19$ ) and neither were their preferred angles (Fig. $5 B$, right; angular-angular correlation test, $\rho=-0.05, p=0.89$ ). Thus, our results support the idea that independent mechanisms are responsible for the local and global motion selectivities in the VPM.

To further investigate how the selectivity to apparent motion might be generated, we compared the response profiles obtained with $\mathrm{C} 2$ caudal deflections to those obtained with the global motion stimuli, all aligned by the onset of the $\mathrm{C} 2$ stimulus (Fig. 5C). For each cell, we first determined the direction among the eight tested that elicited the largest excitatory response component. PSTHs were then averaged across the population $(n=68)$ for this preferred direction and the seven others in $45^{\circ}$ increments. The response profiles all exhibited a large peak $\sim 10 \mathrm{~ms}$ after the onset of $\mathrm{C} 2$ stimulation, followed by a smaller peak $20 \mathrm{~ms}$ later corresponding to the movement of $\mathrm{C} 2$ back to its rest position. Stimulation of $\mathrm{C} 2$ only or of the whole whiskerpad yielded very similar profiles, both in time course and amplitude, for the preferred direction of global motion. Other directions induced a small reduction in amplitude, without changes in the general profile of activity. These results suggest that multiwhisker integration in VPM neurons involves mostly suppressive response interactions among whiskers.

\section{The global motion selectivity of VPM neurons is still present during cortical inactivation}

Overall, combining the results of the excitatory and suppressive response components, we have found a significant direction selectivity for 37 of 73 cells (51\%). This is less than at the cortical stage (70\%) (Jacob et al., 2008), suggesting that the cortical selectivity could be primarily due to the tuning of input from VPM, with further intracortical amplification. However, these two structures are also connected by strong feedback projections (Rouiller and Welker, 2000). The reverse scheme could thus also be true, in which the selectivity of VPM cells would be due to recurrent activity from the cortex. To assess the contribution of corticothalamic projections, we recorded the activity of VPM neurons before and during inactivation of the barrel cortex (Fig. 6A). Figure $6 B$ shows the changes in response of a monovibrissal neuron during such an experiment. This neuron initially displayed a strong global motion selectivity of its early excitatory phasic response for $0^{\circ}$ and adjacent angles, accompanied by an isotropic suppression of activity (Fig. 6B1). Cortical activity was then silenced by surface application of $4 \% \mathrm{Mg}^{2+}$, as verified by the lack of spikes, $15 \mathrm{~min}$ after the first application, recorded by an electrode lowered in infragranular layers of the barrel cortex (Fig. 6B1,B2, insets). Responses of the thalamic neuron were still selective to the global motion angle but to a lesser extent (Fig. $6 \mathrm{B1}$, DI before inactivation $=0.42$; Fig. 6 B2, DI during inactivation $=0.24$ ); note, for example, the appearance of an excitatory component for $225^{\circ}$ in the caudoventral direction. 
A

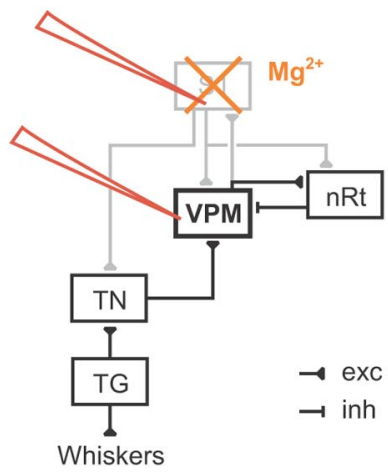

B1

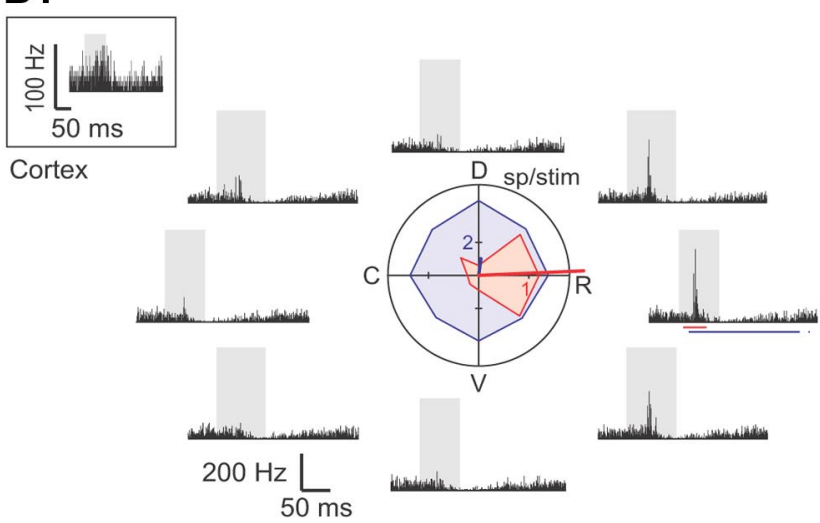

B2
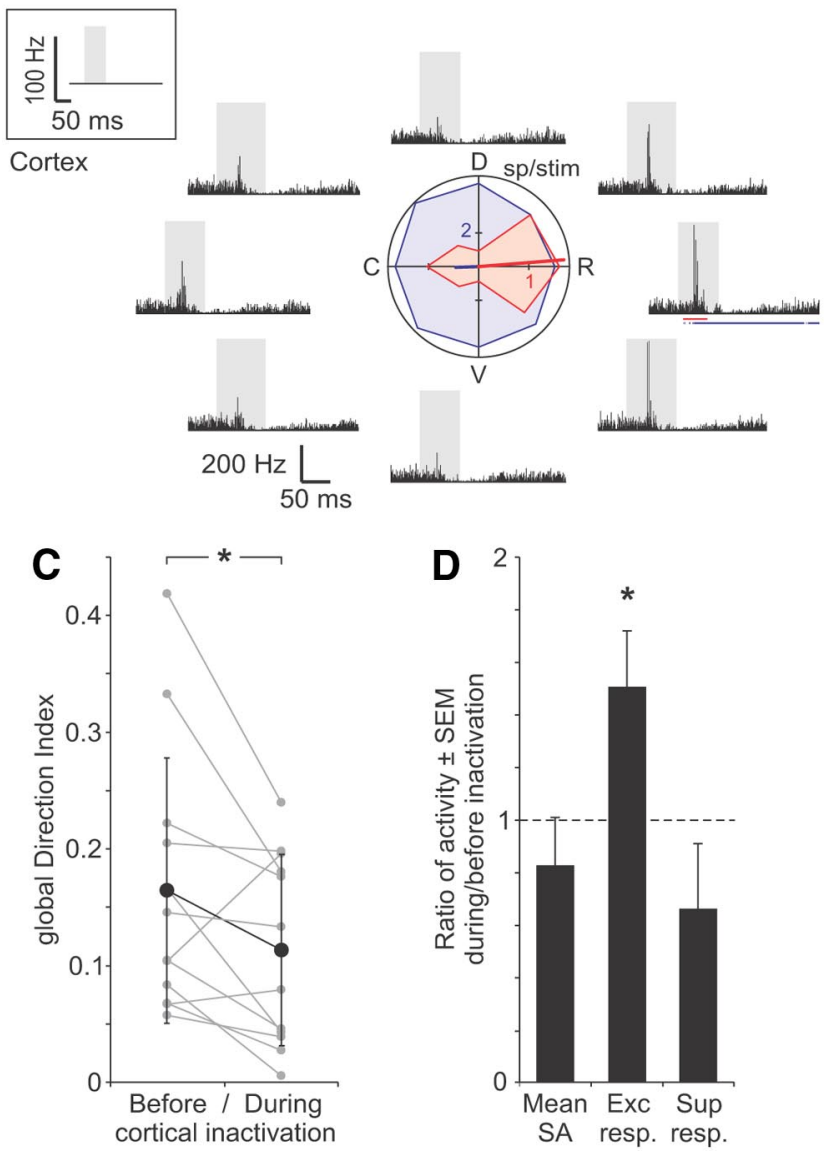

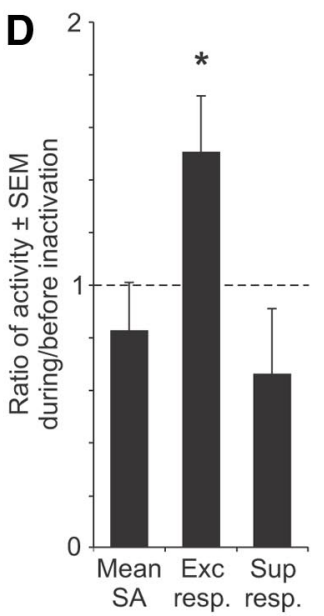

Figure 6. Direction selectivity of VPM neurons for global motion during cortical inactivation. $\boldsymbol{A}$, Schematic diagram of the simultaneous recordings in VPM and barrel cortex infragranular layers while cortical activity was silenced by $\mathrm{Mg}^{2+}$ application. $B$, PSTHs of response and tuning curves for a VPM neuron before (B1) and during (B2) cortical inactivation. Left (insets), PSTHs of

Cortical inactivation was achieved in nine experiments. We report data from 12 cells for which C2 was confirmed to be the functional principal whisker and for which the multiwhisker protocol evoked responses both before and during inactivation. Population analysis indicated a small but significant decrease in the direction selectivity of VPM neurons due to cortical inactivation (Fig. 6C, mean DI before $=0.16$; mean DI during inactivation $=0.11$; Wilcoxon paired sample test, $p=0.03)$. Decreases of the index of selectivity were found in 10 of 12 cases.

We investigated whether the changes in selectivity induced by the removal of activity in the corticothalamic inputs could be linked to other modifications of the response properties of VPM neurons. Spontaneous activity levels were not affected on average (Fig. 6D; Wilcoxon paired sample test, $p=0.27$ ). However, we found a significant increase in the mean amplitude of the excitatory response component (Wilcoxon paired sample test, $p=0.02$ ). The mean amplitude of the suppressive component tended to decrease on average on the five cells which exhibited some suppression initially, but this was not significant (Wilcoxon paired sample test, $p=0.62$ ). In parallel, receptive field estimates showed an increase in the number of whiskers eliciting a significant excitatory response in 4 of 12 cases, while none showed a decrease.

We conclude from these experiments that the selectivity of VPM neurons for global motion is generated subcortically and amplified by the cortical feedback.

\section{Discussion}

We have combined electrophysiological single-unit recording in the VPM nucleus of the thalamus to controlled multiwhisker stimulation generating a global motion across the whiskerpad. Both excitatory and suppressive responses could be elicited, sometimes in the same neuron. Sup-

$\leftarrow$

multiunit cortical activity in infragranular layers during the same multiwhisker stimulation protocols (only one direction of global motion displayed). C, Point-to-point diagram of the Dl before and during cortical inactivation for each neuron studied (gray, $n=12$ ) and mean \pm SD (black). On average, there was a significant decrease of the DI (Wilcoxon paired sample test, $p=0.03$ ). $\boldsymbol{D}$, Average ratio of activity values during cortical inactivation compared with before (mean \pm SEM), for spontaneous activity and for excitatory and suppressive response magnitudes averaged over the eight possible directions of global motion. The excitatory response component increased significantly (Wilcoxon paired sample test, $p=0.02$ ). 
pressive responses were nonselective to the direction of the global stimulus. In contrast, approximately half of the cells exhibiting excitatory responses were directionally selective. Under cortical inactivation, this selectivity decreased on average but remained present. These results suggest that nonlinear processing of stimuli from different whiskers occurs in the VPM even in the absence of corticothalamic feedback, and allows thalamic neurons to extract emergent properties of complex multiwhisker deflections.

Similar results have been previously obtained in the barrel cortex (Jacob et al., 2008). Using the same experimental procedures, we had found that a large proportion of cortical neurons were selective to the direction of global motion. The present results raise the possibility that VPM activity patterns could be responsible for the cortical direction selectivity. However, there were several differences between the two structures. First, whereas preferred directions for global motion showed a bias toward a caudoventral direction in the cortical dataset (Jacob et al., 2008, their Fig. 2D), we found a homogeneous distribution of angles in the VPM (Fig. $2 D$, present study). Interestingly, a similar dissociation between thalamic and cortical directional properties was observed in a recent study (Roy et al., 2011), reporting a bias of thalamocortical inputs along rows in the caudal direction that was absent in the multiunit activity recorded in the thalamus. These and our results suggest that long-range intracortical connections specifically along rows might interact with the thalamocortical input to create this bias.

Second, in the cortex, the preferred directions for global motion of infragranular regular-spiking units were related to the asymmetry angles of their receptive fields, but this did not hold for layer IV units (Jacob et al., 2008). In the thalamus, the characteristics of the receptive fields were independent from the direction selectivity of the cells, thus matching the results for the thalamo-recipient barrel cells.

Finally, the stimulation of remote whiskers was strongly involved in the emergence of direction selectivity of cortical neurons, whereas it had little incidence on the tuning curve of thalamic neurons, for which the proximal periphery was sufficient to express multiwhisker global motion selectivity. Interestingly, it was shown that the presence and amplitude of cross-whisker suppression in a VPM neuron was linearly related to the spatial extent of its dendritic tree in the corresponding neighboring barreloid, thus restricting potential suppressive effects to the principal and four adjacent whiskers (Lavallée and Deschênes, 2004). This is because reticulo-thalamic fibers terminate specifically on distal extra-barreloid dendrites. This result was obtained in experimental conditions in which VPM and nRt neurons were rendered monovibrissal; it remains to be established how cross-whisker excitatory responses in these neurons may modify the nature and extent of this suppression. Nonetheless, it does suggest that VPM multiwhisker processing arises from the proximal periphery.

Together, these discrepancies between the thalamic and cortical direction selectivities suggest that thalamic neurons integrate vibrissal information from a narrower spatial domain than cortical neurons, and point to a probable involvement of both vertical and long-range horizontal intracortical connections in the additional response transformation at the cortical level.

The observed anisotropy of thalamic responses is necessarily the signature of one or several nonlinearities during the transformation of sensory input from vibrissae to VPM. More precisely, nonlinear interactions must exist between responses to different vibrissae. However, anatomical projections along the trigeminothalamo-cortical axis have been found to be remarkably topo-

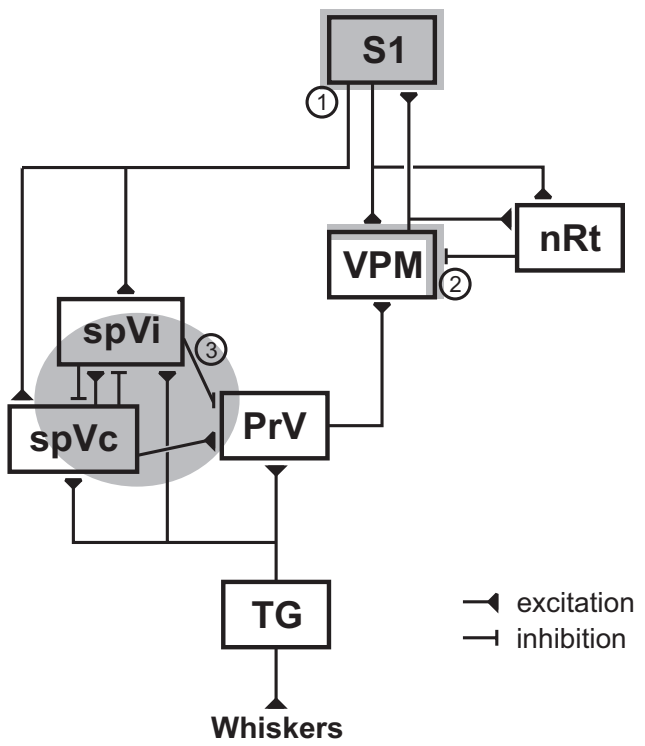

Figure 7. Schematic diagram of the main somatosensory structures and connections proposed to be relevant to the direction selectivity for global motion. TG, Trigeminal ganglion. Other abbreviations are defined in the text; spVi and spVc are the interpolaris and caudalis subdivisions of spV. Gray shading indicates putative sites of cross-whisker information processing that could be responsible for the selectivity to the direction of global motion and that are discussed in the main text. 1, Corticothalamic and corticotrigeminal feedback projections originate from upper layer VI cells that carry substantial cross-whisker information. 2, VPM neurons may receive nonhomologous whisker input from $\mathrm{nRt}$ (and S1). The axons are topographically arranged from one barrel to the corresponding barreloid; the substrate for divergent connections would be the distal dendrites extending outside of the home barreloid of the VPM neuron. 3, Intratrigeminal projections, notably among PrV, spVi, and spVc, carry surround whisker signals. In addition to these three mechanisms, cellular properties (spike threshold, nonlinear summation by the membrane) in PrV and VPM neurons could amplify the direction selectivity for global motion along the pathway.

graphic and parallel, with axonal and dendritic fields often strictly restricted to the small region corresponding to one vibrissa (Deschênes, 2009). This parallel organization strongly limits putative sites of nonlinear processing.

Also, in our study, response profiles when stimulating the whole whiskerpad were similar in magnitude and time course to response profiles when stimulating the principal whisker alone (Hirata and Castro-Alamancos, 2008). Thus, sublinear mechanisms rather than facilitatory ones are likely to be engaged in VPM global motion selectivity, such as those involved in VPM cross-whisker suppression (Simons and Carvell, 1989; Higley and Contreras, 2007). With these constraints in mind, we will consider several possible mechanisms contributing to multiwhisker nonlinear processing in VPM (Fig. 7): (1) feedback from direction-selective cortical neurons, (2) lateral inhibition from the nRt, and (3) multiwhisker processing by the intratrigeminal circuitry.

Cortical feedback (Fig. 7, 1), originating from upper layer VI cells that have large multiwhisker receptive fields (Bourassa et al., 1995; Land et al., 1995), can influence thalamic responses, in particular given the long duration of the multiwhisker protocol. Electrical or chemical stimulation of corticothalamic inputs results in a combination of EPSPs and IPSPs (Landisman and Connors, 2007; Lam and Sherman, 2010), demonstrating direct excitatory effects as well as a disynaptic inhibitory influence through the nRt. Studies using cortical inactivation concluded on either no effect of the cortical feedback on VPM responses (Diamond et al., 1992a; Higley and Contreras, 2007; Roy et al., 2011), 
facilitatory effects (Yuan et al., 1985, 1986), or more complex modifications (Ghazanfar et al., 2001). Of particular relevance to our study, electrical stimulation results in net facilitatory effects of sensory responses when activating the cortical barrel corresponding to the barreloid recorded from (Temereanca and Simons, 2004), but only for whisker deflections in the preferred direction of the cortical stimulation site (Li and Ebner, 2007), revealing a high corticothalamic feedback specificity. In this framework, we tested whether the VPM selectivity for global motion could be partly or entirely explained by the cortical input. Silencing of the barrel cortex modified but did not abolish direction selectivity in the thalamus. Evidence of nonlinear processing of multiwhisker stimuli was still present after prolonged periods of barrel cortex inactivation, indicating that the subcortical network is already capable of extracting higher order features from an otherwise locally invariant stimulus.

Our second hypothesis was that intrathalamic processing by the thalamoreticular loop might differentially suppress responses to nonpreferred directions (Fig. 7, 2). As previously mentioned in a few studies (Shosaku, 1985; Simons and Carvell, 1989; Minnery et al., 2003), we observed many cases of evoked suppression of activity in VPM neurons. Because no intrinsic inhibitory interneurons are present in the VPM, the suppressive components of responses most probably result from inhibitory projections from nRt (Lee et al., 1994; Brecht and Sakmann, 2002; CastroAlamancos, 2002b) known to mediate information from the principal and immediately adjacent whiskers (Lavallée and Deschênes, 2004; Pinault, 2004). However, in our study, suppression of activity was not selective to the direction of apparent motion of the multiwhisker stimulus, implying that the nRt inhibitory feedback cannot explain the emergence of global motion selectivity of VPM responses.

The third possibility that we considered is that complex features of multiwhisker stimuli are already extracted by the trigeminal circuitry (Fig. 7, 3). PrV neurons projecting to VPM have receptive fields composed of a principal whisker, inherited from direct trigeminal ganglion afferents (Henderson and Jacquin, 1995; Veinante and Deschênes, 1999), and several surround whiskers, for which the evoked activity is dependent on intratrigeminal connections between $\operatorname{PrV}$ and the spinal trigeminal nucleus $(\mathrm{spV})$. In particular, these cross-whisker responses would rely heavily on excitatory inputs to $\operatorname{PrV}$ from the caudal subnucleus $(\mathrm{spVc})$ and on inhibitory modulation by the interpolar subnucleus (spVi) (Furuta et al., 2008). The spVi modulation would contribute to sharpen directional tuning (Bellavance et al., 2010) and generate detectable cross-whisker suppression already in $\mathrm{PrV}$ (Minnery and Simons, 2003; Timofeeva et al., 2004). It would be most interesting to record in the trigeminal nuclei to establish whether selectivity to global motion is already present at that stage. Given the comparisons of cross-whisker suppression in PrV and VPM (Minnery et al., 2003; Higley and Contreras, 2007), we anticipate that direction selectivity to global motion would be found in the PrV output, although at a lesser degree than in the VPM. Intrinsic nonlinearities of VPM cells, such as the spike threshold and the nonlinear summation of inputs by the membrane, could amplify the anisotropy of PrV multiwhisker responses.

The main conclusion of our study is that substantial nonlinear processing takes place already at subcortical stages, enabling the extraction of global features of a complex multiwhisker stimulus before reaching the cortex. Evidence for the contribution of several levels of sensory processing in the detection of specific features has been accumulating in the vibrissal system, concerning adaptation to high-frequency stimuli (Ganmor et al., 2010), cross-whisker suppression (Higley and Contreras, 2007), and directional tuning (Lichtenstein et al., 1990; Minnery et al., 2003; Timofeeva et al., 2003; Wilent and Contreras, 2005). Each level (trigeminal, thalamic, cortical) is a mixed excitation-inhibition network. This distributed organization in a multilevel balanced system provides a large flexibility, as the combination of influences on each substructure renders the potential modifications of sensory processing extremely diverse, allowing to be best adapted to a particular behavioral and sensory context. In this view of distributed sensory processing, it is not surprising to discover that subcortical stages are not mere relay stations but already extract relevant features of sensory stimuli.

\section{References}

Aguilar JR, Castro-Alamancos MA (2005) Spatiotemporal gating of sensory inputs in thalamus during quiescent and activated states. J Neurosci 25:10990-11002.

Andermann ML, Moore CI (2006) A somatotopic map of vibrissa motion direction within a barrel column. Nat Neurosci 9:543-551.

Armstrong-James M, Callahan CA (1991) Thalamo-cortical processing of vibrissal information in the rat. II. Spatiotemporal convergence in the thalamic ventroposterior medial nucleus (VPm) and its relevance to generation of receptive fields of S1 cortical "barrel” neurones. J Comp Neurol 303:211-224.

Arsenault D, Zhang ZW (2006) Developmental remodelling of the lemniscal synapse in the ventral basal thalamus of the mouse. J Physiol 573:121-132.

Bellavance MA, Demers M, Deschênes M (2010) Feedforward inhibition determines the angular tuning of vibrissal responses in the principal trigeminal nucleus. J Neurosci 30:1057-1063.

Bourassa J, Pinault D, Deschênes M (1995) Corticothalamic projections from the cortical barrel field to the somatosensory thalamus in rats: a single-fibre study using biocytin as an anterograde tracer. Eur J Neurosci 7:19-30.

Brecht M, Sakmann B (2002) Whisker maps of neuronal subclasses of the rat ventral posterior medial thalamus, identified by whole-cell voltage recording and morphological reconstruction. J Physiol 538:495-515.

Carvell GE, Simons DJ (1995) Task- and subject-related differences in sensorimotor behavior during active touch. Somatosens Mot Res 12:1-9.

Castro-Alamancos MA (2002a) Properties of primary sensory (lemniscal) synapses in the ventrobasal thalamus and the relay of high-frequency sensory inputs. J Neurophysiol 87:946-953.

Castro-Alamancos MA (2002b) Different temporal processing of sensory inputs in the rat thalamus during quiescent and information processing states in vivo. J Physiol 539:567-578.

Chapin JK, Lin CS (1984) Mapping the body representation in the SI cortex of anesthetized and awake rats. J Comp Neurol 229:199-213.

Chiaia NL, Rhoades RW, Fish SE, Killackey HP (1991) Thalamic processing of vibrissal information in the rat: II. Morphological and functional properties of medial ventral posterior nucleus and posterior nucleus neurons. J Comp Neurol 314:217-236.

Deschênes M (2009) Vibrissal afferents from trigeminus to cortices. Scholarpedia 4:7454.

Deschênes M, Timofeeva E, Lavallée P (2003) The relay of high-frequency sensory signals in the whisker-to-barreloid pathway. J Neurosci 23:6778-6787.

Diamond ME, Armstrong-James M, Budway MJ, Ebner FF (1992a) Somatic sensory responses in the rostral sector of the posterior group (POm) and in the ventral posterior medial nucleus (VPM) of the rat thalamus: dependence on the barrel field cortex. J Comp Neurol 319:66-84.

Diamond ME, Armstrong-James M, Ebner FF (1992b) Somatic sensory responses in the rostral sector of the posterior group (POm) and in the ventral posterior medial nucleus (VPM) of the rat thalamus. J Comp Neurol 318:462-476.

Fisher NI (1996) Statistical analysis of circular data. Cambridge, UK: Cambridge UP.

Friedberg MH, Lee SM, Ebner FF (1999) Modulation of receptive field properties of thalamic somatosensory neurons by the depth of anesthesia. J Neurophysiol 81:2243-2252. 
Furuta T, Timofeeva E, Nakamura K, Okamoto-Furuta K, Togo M, Kaneko T, Deschênes M (2008) Inhibitory gating of vibrissal inputs in the brainstem. J Neurosci 28:1789-1797.

Ganmor E, Katz Y, Lampl I (2010) Intensity-dependent adaptation of cortical and thalamic neurons is controlled by brainstem circuits of the sensory pathway. Neuron $66: 273-286$.

Ghazanfar AA, Krupa DJ, Nicolelis MA (2001) Role of cortical feedback in the receptive field structure and nonlinear response properties of somatosensory thalamic neurons. Exp Brain Res 141:88-100.

Henderson TA, Jacquin MF (1995) What makes subcortical barrels: requisite trigeminal circuitry and developmental mechanisms. In: The barrel cortex of rodents, cerebral cortex, Vol 11 (Jones EG, Diamond IT, eds), pp 123-187. New York: Springer.

Higley MJ, Contreras D (2007) Cellular mechanisms of suppressive interactions between somatosensory responses in vivo. J Neurophysiol 97:647658.

Hirata A, Castro-Alamancos MA (2008) Cortical transformation of widefield (multiwhisker) sensory responses. J Neurophysiol 100:358-370.

Ito M (1988) Response properties and topography of vibrissa-sensitive VPM neurons in the rat. J Neurophysiol 60:1181-1197.

Jacob V, Le Cam J, Ego-Stengel V, Shulz DE (2008) Emergent properties of tactile scenes selectively activate barrel cortex neurons. Neuron 60:11121125.

Jacob V, Estebanez L, Le Cam J, Tiercelin JY, Parra P, Parésys G, Shulz DE (2010) The matrix: a new tool for probing the whisker-to-barrel system with natural stimuli. J Neurosci Methods 189:65-74.

Knutsen PM, Pietr M, Ahissar E (2006) Haptic object localization in the vibrissal system: behavior and performance. J Neurosci 26:8451-8464.

Kremer Y, Léger JF, Goodman D, Brette R, Bourdieu L (2011) Late emergence of the vibrissa direction selectivity map in the rat barrel cortex. J Neurosci 31:10689-10700.

Krupa DJ, Matell MS, Brisben AJ, Oliveira LM, Nicolelis MA (2001) Behavioral properties of the trigeminal somatosensory system in rats performing whisker-dependent tactile discriminations. J Neurosci 21:5752-5763.

Lam YW, Sherman SM (2010) Functional organization of the somatosensory cortical layer 6 feedback to the thalamus. Cereb Cortex 20:13-24.

Land PW, Buffer SA Jr, Yaskosky JD (1995) Barreloids in adult rat thalamus: three-dimensional architecture and relationship to somatosensory cortical barrels. J Comp Neurol 355:573-588.

Landisman CE, Connors BW (2007) VPM and PoM nuclei of the rat somatosensory thalamus: intrinsic neuronal properties and corticothalamic feedback. Cereb Cortex 17:2853-2865.

Lavallée P, Deschênes M (2004) Dendroarchitecture and lateral inhibition in thalamic barreloids. J Neurosci 24:6098-6105.

Le Cam J, Estebanez L, Jacob V, Shulz DE (2011) The spatial structure of multi-whisker receptive fields in the barrel cortex is stimulus-dependent. J Neurophysiol 106:986-998.

Lee SM, Friedberg MH, Ebner FF (1994) The role of GABA-mediated inhibition in the rat ventral posterior medial thalamus. I. Assessment of receptive field changes following thalamic reticular nucleus lesions. J Neurophysiol 71:1702-1715.

Legéndy CR, Salcman M (1985) Bursts and recurrences of bursts in the spike trains of spontaneously active striate cortex neurons. J Neurophysiol 53:926-939.

Li L, Ebner FF (2007) Cortical modulation of spatial and angular tuning maps in the rat thalamus. J Neurosci 27:167-179.
Lichtenstein SH, Carvell GE, Simons DJ (1990) Responses of rat trigeminal ganglion neurons to movements of vibrissae in different directions. Somatosens Mot Res 7:47-65.

Minnery BS, Simons DJ (2003) Response properties of whisker-associated trigeminothalamic neurons in rat nucleus principalis. J Neurophysiol 89:40-56.

Minnery BS, Bruno RM, Simons DJ (2003) Response transformation and receptive-field synthesis in the lemniscal trigeminothalamic circuit. J Neurophysiol 90:1556-1570.

Nicolelis MA, Chapin JK (1994) Spatiotemporal structure of somatosensory responses of many-neuron ensembles in the rat ventral posterior medial nucleus of the thalamus. J Neurosci 14:3511-3532.

Paxinos G, Watson C (2007) The rat brain in stereotaxic coordinates, Ed 6. London, UK: Academic.

Pinault D (2004) The thalamic reticular nucleus: structure, function and concept. Brain Res Brain Res Rev 46:1-31.

Rouiller EM, Welker E (2000) A comparative analysis of the morphology of corticothalamic projections in mammals. Brain Res Bull 53:727-741.

Roy NC, Bessaih T, Contreras D (2011) Comprehensive mapping of whisker-evoked responses reveals broad, sharply tuned thalamocortical input to layer 4 of barrel cortex. J Neurophysiol 105:2421-2437.

Shosaku A (1985) A comparison of receptive field properties of vibrissa neurons between the rat thalamic reticular and ventro-basal nuclei. Brain Res 347:36-40.

Simons DJ, Carvell GE (1989) Thalamocortical response transformation in the rat vibrissa/barrel system. J Neurophysiol 61:311-330.

Swindale NV (1998) Orientation tuning curves: empirical description and estimation of parameters. Biol Cybern 78:45-56.

Temereanca S, Simons DJ (2004) Functional topography of corticothalamic feedback enhances thalamic spatial response tuning in the somatosensory whisker/barrel system. Neuron 41:639-651.

Timofeeva E, Mérette C, Emond C, Lavallée P, Deschênes M (2003) A map of angular tuning preference in thalamic barreloids. J Neurosci 23: 10717-10723

Timofeeva E, Lavallée P, Arsenault D, Deschênes M (2004) Synthesis of multiwhisker-receptive fields in subcortical stations of the vibrissa system. J Neurophysiol 91:1510-1515.

Van Der Loos H (1976) Barreloids in mouse somatosensory thalamus. Neurosci Lett 2:1-6.

Veinante P, Deschênes M (1999) Single- and multi-whisker channels in the ascending projections from the principal trigeminal nucleus in the rat. J Neurosci 19:5085-5095.

Waite PM (1973) The responses of cells in the rat thalamus to mechanical movements of the whiskers. J Physiol 228:541-561.

Wilent WB, Contreras D (2005) Dynamics of excitation and inhibition underlying stimulus selectivity in rat somatosensory cortex. Nat Neurosci $8: 1364-1370$.

Williams MN, Zahm DS, Jacquin MF (1994) Differential foci and synaptic organization of the principal and spinal trigeminal projections to the thalamus in the rat. Eur J Neurosci 6:429-453.

Yuan B, Morrow TJ, Casey KL (1985) Responsiveness of ventrobasal thalamic neurons after suppression of S1 cortex in the anesthetized rat. J Neurosci 5:2971-2978.

Yuan B, Morrow TJ, Casey KL (1986) Corticofugal influences of S1 cortex on ventrobasal thalamic neurons in the awake rat. J Neurosci 6:36113617 . 